\title{
Nota Farmacológica: Terapia basada en la incretina: una nueva propuesta en el tratamiento de la Diabetes Mellitus tipo 2
}

\author{
Incretin-based therapy: a new alternative for the treatment of Type 2 Diabetes Mellitus
}

Maia Burstein*

\begin{abstract}
Resumen
Los tratamientos para la diabetes tipo 2 suelen ser menos efectivos a lo largo del tiempo, tanto por una progresiva falla de la secreción de insulina por las células beta, como por diversos efectos adversos. En la presente nota se describe un grupo de nuevos fármacos que actúan a través de la via de la incretina, tanto los incretina-miméticos (análogos del péptido glucagon símil-1[GLP-1]) como el exenatide, como los inhibidores de la enzima que degrada la incretina, la dipeptidilpeptidasa-4 (DPP4). Ellos se indican cuando no se logra buen control glucémico a pesar de un tratamiento adecuado combinado de sulfonilureas y metformina. Las conclusiones de una reciente revisión sistemática son detalladas. Hacen falta estudios de farmacovigilancia adecuados de efectos adversos, especialmente entre los inhibidores de la DPP4, y estudios a más largo plazo, para determinar el rol de esta nueva clase de agentes en la diabetes tipo 2.

\section{Abstract}

Type 2 diabetes treatments have a decreasing effectiveness with time, both due to a progressive insulin secretion failure of beta cells and to different adverse effects. In the present article a new group of drugs that act through the incretin, both the incretin mimetics (glucagonlike peptide 1 [GLP-1] analogs) like exenatide, as well as dipeptidyl peptidase 4 (DPP4) inhibitors. They are used when there is no adequate glycemic control in spite of using recommended doses of a combination therapy of sulfonylureas and metformin. The conclusions of a recent systematic review are detailed. Careful postmarketing surveillance for adverse effects, especially among the DPP4 inhibitors, and longer-term studies to determine the role of this new drug class for type 2 diabetes.
\end{abstract}

Burstein M. Terapia basada en la incretina: una nueva propuesta en el tratamiento de la Diabetes Mellitus tipo 2. Evid. actual. práct. ambul; 11(1): 2829, ene-feb.2008.

\section{Introducción}

En muchos de los individuos con diabetes tipo 2 la hiperglucemia es causada por falla en la capacidad secretora de insulina de las células beta. Esta falla en general progresa a pesar de un buen tratamiento con dieta, metformina, sulfonilureas (SU) o insulina. Varios de los tratamientos tradicionales suelen verse limitados por efectos adversos, como el aumento de peso, la hipoglucemia, intolerancia gastrointestinal y edema periférico. La adición de tiazolidinedionas o insulina a la terapia combinada puede asociarse al aumento de los efectos adversos como la ganancia de peso.

En los últimos años se ha introducido un nuevo grupo de fármacos que actúan sobre la vía de la incretina, uno de los factores reguladores de la secreción de insulina. Se denomina efecto incretina al efecto de la glucosa oral sobre la secreción de insulina y está mediada por péptidos producidos en células del intestino delgado. El más conocido de ellos es el péptido glucagon símil-1 (GPL-1).

Dentro del mencionado grupo de fármacos se encuentran los incretina miméticos (análogos del péptido glucagon símil-1 [GPL-1]), entre los cuales el más conocido es el exenatide (exedin-4) y los inhibidores de la enzima que degrada la incretina, la dipeptidilpeptidasa-4 (DPP4), siendo el más conocido la sitagliptina. Estos fármacos reemplazan el efecto de la glucosa gastrointestinal típicamente perdido en los individuos con diabetes tipo 2 . Sus efectos sobre el metabolismo glucémico son diversos: estimulan la secreción de la insulina de las células beta cuando la glucosa de la sangre está elevada; restauran la primera respuesta de la insulina, que típicamente se pierde en la diabetes tipo 2; así como también la segunda fase; reducen la producción de glucagon postprandial; retardan el vaciamiento gástrico y reducen la ingesta de alimentos.

Los incretina miméticos e inhibidores de la DPP4 están indicados cuando no se logra buen control glucémico a pesar de un tratamiento adecuado combinado de SU y metformina. El exenatide es un péptido de 39 aminoácidos incretina mimético que se administra en forma subcutánea, 15 minutos antes de las comidas.

La aparente ventaja de este nuevo grupo es que mejoran el control glucémico, retardando el deterioro de las células $\beta$ del pán- creas, mejoran el control sobre la glucemia postprandial y el peso corporal. Recientemente se ha publicado un metaanálisis ${ }^{1}$ sobre la evidencia disponible de la efectividad y seguridad de las terapias sobre la incretina en pacientes con diabetes tipo 2 . Los principales puntos a evaluar fueron los efectos sobre la glucemia en ayunas y la postprandial, los cambios de la hemoglobina glicosilada ( $\mathrm{Hb} \mathrm{A1c}$ ) respecto de la inicial y el porcentaje de individuos que llegan a una meta de $\mathrm{HbA} 1 \mathrm{c}$ menor de $7 \%$. Además se evaluó la variación del peso corporal y del perfil lipídico. En cuanto a la seguridad se extrajo información sobre incidencia de hipoglucemia y eventos adversos totales.

Se incluyeron 29 ensayos clínicos, de los que sólo tres tenían una duración mayor de 30 semanas. Se agruparon ocho ensayos sobre análogos de la GLP-1 adicionados a terapéuticas con hipoglucemiantes orales y conductuales, comparados con placebo, metformina o insulina. Incluyeron un total de 3.139 pacientes de edades de 19 a 78 años. Por otro lado, se incluyeron 13 ensayos doble ciego que compararon inhibidores de DPP4 como monoterapia o adicionada a hipoglucemiantes orales o insulina (con un total de 4.780 pacientes). Cuatro ensayos ( $n=3.053$ ) compararon directamente a los inhibidores de DPP4 con hipoglucemiantes orales.

\section{Incretina miméticos (análogos GLP-1)}

Se hallaron diferencias significativas entre los análogos GLP-1 y placebo en cuanto a la reducción de HbA1c $(-0,97 \%$ IC95\% $-1,13$ a $-0,81 \%)$. En estudios en que se comparó el exenatide con insulina glargina o aspártica bifásica, no se hallaron diferencias significativas. Además, los pacientes tratados con exenatide presentaron mayores chances de lograr $\mathrm{HbA} 1 \mathrm{c}$ de $7 \% 0$ menor que aquellos con placebo ( $45 \%$ vs $10 \%$, respectivamente, RR 4,2, IC95\% 3,2 a 5,5) y tampoco hubo diferencias significativas al comparar exenatide con insulina.

En cuanto a la glucemia en ayunas y postprandial, se halló una reducción de la glucemia en ayunas significativa con comparado con placebo (-27mg/dL IC $95 \%-33$ a $-21 \mathrm{mg} / \mathrm{dL})$. Al comparar los efectos del exenatide vs insulina, se encontró diferencia significativa a favor de los análogos GLP-1 con respecto a la glucemia postprandial, pero no a la glucemia en ayunas.

* Servicio de Medicina Familiar y Preventiva. Hospital Italiano de Buenos Aires 
Para la glucemia postprandial agregado a la dieta general, el exenatide mostró un efecto dosis dependiente.

Respecto del peso corporal, se encontró una reducción significativa del peso en pacientes con análogos GLP-1 vs los comparadores (-2,7kg IC95\% -3,95 a -0,78) y esta diferencia resultó mayor entre exenatide y la insulina. Se observó con el exenatide una pérdida de peso progresiva, dosis dependiente y sin meseta aparente a las 30 semanas. Esta reducción no pareció asociarse a la presencia de náuseas. No se observaron efectos significativos sobre el perfil lipídico.

Los principales eventos adversos reportados fueron hipoglucemia y náuseas. Se reportaron sólo cinco casos de hipoglucemia severa entre 2.781 pacientes tratados con exenatide, todos ellos recibían además SU. La incidencia de hipoglucemia leve a moderada fue mayor en pacientes con exenatide que con placebo ( $16 \%$ vs $7 \%$, RR 2,3 IC95\% 1,1 a $4,9)$, especialmente si se asociaba a SU. Estos eventos fueron más frecuentes al inicio del tratamiento con posterior declinación. El riesgo fue similar al comparar exenatide con insulina. La incidencia de náuseas y vómitos fue mayor en el grupo exenatide ( $57 \%$ de pacientes con náuseas y $17 \%$ con vómitos), más frecuentes durante las primeras ocho semanas de tratamiento. También se observó aumento de la incidencia de diarrea. En general, los efectos adversos gastrointestinales llevaron a una mayor tasa de abandono que en el grupo placebo. El liraglutide, otro análogo GLP-1, no se asoció a náuseas ni vómitos.

\section{Inhibidores de DPP4 (estimuladores de la incretina)}

Se incluyeron las dos drogas disponibles: sitagliptina y vidagliptina. Ambas mostraron reducir la $\mathrm{HbA1c}$ comparadas con placebo $(-0,74 \%$ IC $95 \%-0,85$ a $-0,62 \%)$, tanto adicionadas a otros hipoglucemiantes o como monoterapia. Los inhibidores DPP4 fueron levemente menos efectivos al compararlos con otros hipoglucemiantes $(0,21 \% ; \quad I C 95 \% \quad 0,02 \%$ a $0.39 \%)$. Ambas drogas mostraron una significativa reducción de la glucemia postprandial a las 2 y 4 hs, especialmente a altas dosis. Respecto del peso corporal, se observó un leve aumento comparado con placebo $(0,5 \mathrm{~kg} I \mathrm{C} 95 \%$ 0,3 a $0,7 \mathrm{~kg})$. En ensayos de no inferioridad mostraron resultados favorables comparados con glipizida y tiazolidinedionas pero no con metformina. Aunque no se hallaron efectos consistentes sobre el perfil lipídico, se reportó una leve mejoría en el nivel de triglicéridos, LDL y HDL-colesterol.

No se hallaron diferencias en la incidencia de hipoglucemia respecto de los grupos de comparación pero sí mayor incidencia de nasofaringitis, infecciones urinarias y cefaleas. De todos modos, pero para conocer su real importancia, se requieren estudios de mayor seguimiento y cantidad de pacientes.

\section{Conclusiones}

La inclusión del grupo de medicamentos que actúan sobre la vía de la incretina parece ser el comienzo de una interesante opción terapéutica recientemente conocida y que podría llegar a ser un adelanto significativo. Se estima que sus principales ventajas se basan en que su efecto, basado en el efecto de la glucosa gastrointestinal sobre la secreción de insulina, interviene sobre el control de la glucemia de modo más fisiológico y eficaz que las demás drogas, ya que restablece un mecanismo de homeostasis perdido en los diabéticos tipo 2. Por ello, se espera que la terapia basada en incretina logre un mayor control de la glucemia postprandial y un retardo en la declinación de la función de las células beta pancreáticas, y de ese modo mejorar el pronóstico de la diabetes a largo plazo. El efecto sobre el peso corporal de los análogos del GLP-1 es un dato adicional de importancia ya que ayuda a optimizar el perfil metabólico y mejora la adherencia.

La evidencia con la que contamos es aún limitada ya que la mayoría de los estudios tuvieron un breve seguimiento, y son muy escasos los que llegan a las 30 semanas. En nuestro medio el exenatide cuenta con dos desventajas significativas: la administración por vía parenteral y su difícil acceso por costo y disponibilidad. También son insuficientes los estudios que comparan la adición de exenatide vs insulina a esquemas terapéuticos más frecuentes (principalmente metformina) para determinar el beneficio de elegir exenatide. Es de esperar que se aguarde a contar con evidencia mucho más contundente, así como una prolija valoración de la relación de costo-efectividad para que los médicos la incorporen a sus indicaciones habituales.

Además, poco sabemos aún sobre su utilidad en pacientes que inician su tratamiento de diabetes y su real efecto sobre la progresión de la diabetes hacia el requerimiento de insulinoterapia. Para ello se requieren estudios de comparación directa con hipoglucemiantes orales tradicionales y estudios de seguimiento mucho más prolongados de los que contamos hasta el momento. Cabe suponer que se dedicarán importantes esfuerzos a desarrollar nuevas drogas en la misma familia y que en un futuro cercano contemos con mayor evidencia de su utilidad.

Concluyendo, la terapia basada en la incretina ofrece una opción alternativaa los hipoglucemiantes actualmente disponibles en adultos (no en embarazadas) con una eficacia modesta. Hacen falta estudios de farmacovigilancia adecuados, especialmente entre los inhibidores de la DPP4, y estudios a más largo plazo, para determinar el rol de esta nueva clase de agentes en la diabetes tipo 2.

Fuente principal

1. Renee E Amori y col, Efficacy and Safety of Incretin Therapy in Type 2 Diabetes, Systematic Review and Meta-analysis. JAMA. 2007 Jul 11;298(2):194-206 